\title{
Yaw Motion Control of Electromagnetic Guidance System for High-Speed Maglev Vehicles
}

\author{
Chang-Hyun Kim ${ }^{\dagger}$, Chang-Wan Ha*, Jaewon Lim*, Hyung-Suk Han* and Ki-Jung Kim**
}

\begin{abstract}
In an attractive-type high-speed magnetic levitation (maglev) train, separate electromagnets are used for lateral guidance. For the guidance system, a U-shaped electromagnet that uses transverse flux has been designed and analyzed. Electromagnets counterbalance centrifugal forces during curving as well as under all operating conditions so that the clearances between on-board magnets and the guideway surface can be maintained within an allowable range. Because of the rigidity of the bogie, coupling effects exist among the magnets that are controlled independently. This study incorporates bogie yaw motion into control law for adjusting the voltages applied to magnets to provide constant clearance control. The effects of bogie yaw motion on guidance control performance are investigated experimentally using a full-scale vehicle.
\end{abstract}

Keywords: Air gap, Control law, Guidance control, Maglev vehicle, Yaw motion

\section{Introduction}

Magnetic levitation is a promising technology for highspeed transportation systems in which speed reaches 550 $\mathrm{km} / \mathrm{h}$ as a result of its non-contact operation. The previous low-speed $(110 \mathrm{~km} / \mathrm{h})$ maglev train named "EcoBee" in Korea uses attractive-type electromagnetic suspension (EMS) and linear induction motor (LIM) propulsion. Because of low energy efficiency that originates from drag force and leakage inductance, LIM is not suitable for high-speed operation. A linear synchronous motor (LSM) propulsion has been adopted for existing high-speed maglev trains such as the German Transrapid and Japanese MLX [1]. The Korean high-speed maglev train under development employs attractive EMS and LSM propulsion [2]. EcoBee uses a lateral component of the levitation force as a guidance force, whereas the high-speed maglev adopts separate guidance electromagnets to ensure lateral riding stability.

The lateral air gap between electromagnets and the guideway should be controlled within an allowable range to avoid collision. In previous systems, each guidance magnet is controlled independently because it is implemented with simple hardware [3, 4]. Main disturbances of the guidance system derive from irregularities of reaction plates and centrifugal forces when curving. Because of the rigidity of the bogie, coupling effects exist among the independently controlled magnets. To improve ride quality

$\dagger$ Corresponding Author: Dept. of Magnetic Levitation and Linear Drive, Korea Institute of Machinery and Materials, Korea.

(chkim78@kimm.re.kr)

* Dept. of Magnetic Levitation and Linear Drive, Korea Institute of Machinery and Materials, Korea. (\{hawan, einses, hshan\} @.kimm.re.kr)

** Research Institute for High-Technology Transportation, Chungnam National University, Korea. (ready2go@cnu.ac.kr)

Received: February 23, 2016; Accepted: May 5, 2016 and reduce the effect of coupling, bogie yaw motion is considered in control law and an additional control is applied to magnets to provide a constant yaw angle.

To address these needs, we developed an electromagnetic guidance system for a high-speed maglev vehicle. The guidance electromagnet and yaw motion controller have been designed and analyzed. The effects of yaw motion on guidance control are investigated experimentally using a full-scale maglev test vehicle.

\section{Electromagnetic Guidance System Design for High-Speed Maglev Vehicle}

\subsection{High-speed maglev test vehicle}

The full-scale attractive-type high-speed maglev test

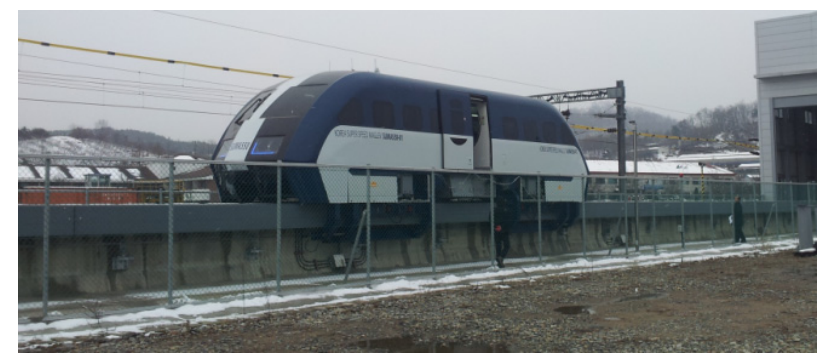

Fig. 1. Full-scale attractive-type high-speed maglev test vehicle

Table 1. Specifications of high-speed maglev test vehicle

\begin{tabular}{c|c}
\hline Items & Values \\
\hline Maximum design speed & $550 \mathrm{~km} / \mathrm{h}$ \\
\hline Carbody mass & $12,576 \mathrm{~kg}$ \\
\hline Bogie mass & $8,400 \mathrm{~kg}$ \\
\hline
\end{tabular}




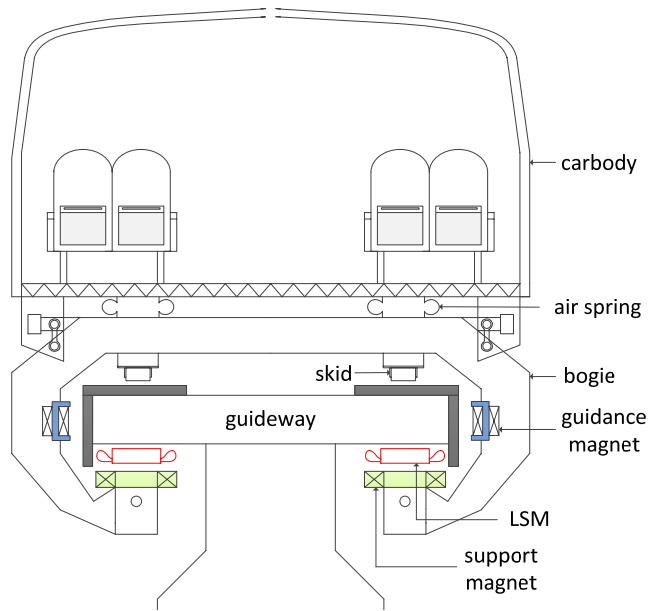

(a)

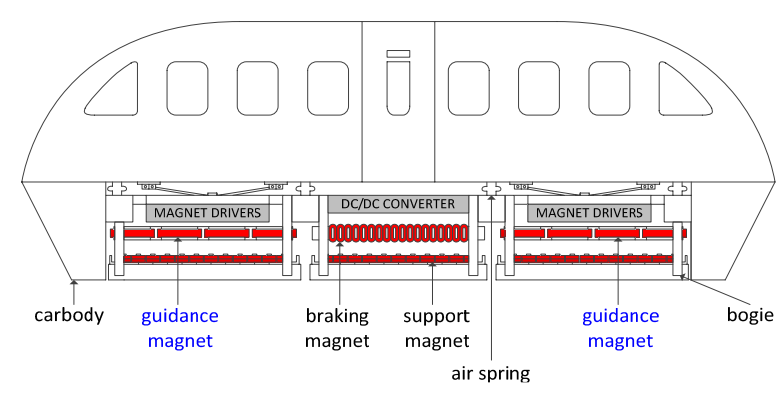

(b)

Fig. 2. (a) Cross section of high-speed maglev test vehicle and (b) Two bogie module articulated vehicle

vehicle and its $150 \mathrm{~m}$ test track are shown in Fig. 1. The major specifications of the maglev test vehicle are shown in Table 1. The guideway is surrounded by bogie structures, and the configuration of the bogie of the maglev test vehicle is shown in Fig. 2. Two bogies exist in front and rear sections of the test vehicle and guidance magnets are attached on both sides of the bogies. Braking magnets are installed instead of guidance magnets between the two bogies. Each bogie has two sensor modules and eight guidance magnets (four magnets on each side). The signals from two sensor modules are shared by each control hardware (magnet driver). A sensor module consists of two gap sensors and an accelerometer. The guidance magnets are divided into four sections according to location (right/left, front/rear). We can essentially control each section independently [5].

\subsection{Electromagnetic guidance system design}

Fig. 3 shows the three-dimensional (3D) view of guidance magnet and magnetic flux distribution. Guidance magnets use transverse magnetic flux perpendicular to the moving direction to minimize the magnetic drag force. The required force is determined based on total weight, speed, curve radius, and so on. We set the required force as $30 \%$
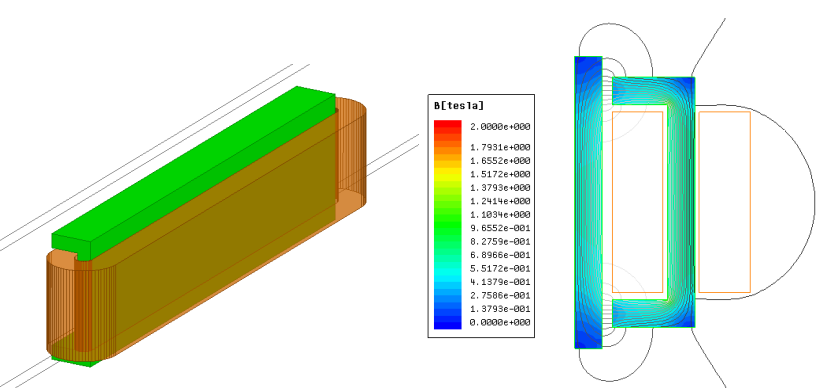

Fig. 3. Three-dimensional view of guidance magnet and magnetic flux distribution

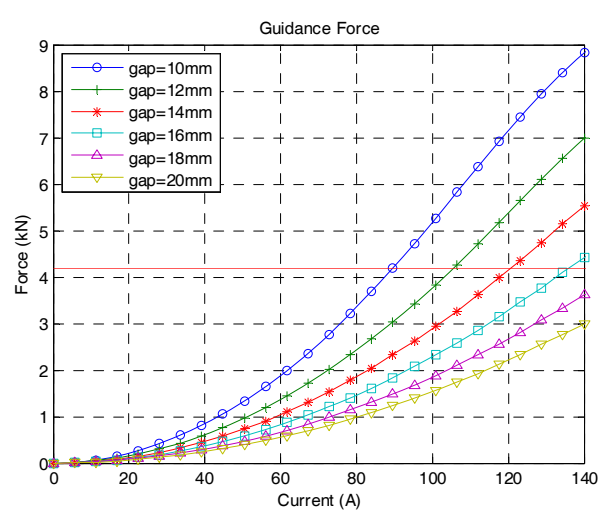

Fig. 4. Attraction force characteristics based on air gap and current

Table 2. Main specifications of the guidance magnet

\begin{tabular}{c|c}
\hline Items & Values \\
\hline Required force per magnet & $4.2 \mathrm{kN}$ \\
\hline Nominal airgap & $10 \mathrm{~mm}$ \\
\hline Pole area & $0.012 \mathrm{~m}^{3}$ \\
\hline Coil turns & 120 \\
\hline Coil material & Aluminum \\
\hline Guidance rail thickness & $20 \mathrm{~mm}$ \\
\hline
\end{tabular}

of the total weight. The designed parameters are listed in Table 2.

In the design process, many aspects such as magnetic saturation, air gap, thickness of the reaction plate, inductance, and lateral separation are considered. The final design is verified using 2D/3D finite element analysis tools as shown in Fig. 3. The magnetic leakage flux is relatively small and reduced magnetic flux saturation is observed. Attraction force characteristics based on air gap and current are shown in Fig. 4. The generated guidance force at a 10 $\mathrm{mm}$ air gap is $4.2 \mathrm{kN}$ when the coil current is $90 \mathrm{~A}$.

The guidance force, $F$, is characterized as a nonlinear function of an air gap, $c$, and a coil current, $i$.

$$
F(i(t), c(t))=\frac{\mu_{0} N^{2} A}{4} \frac{i(t)^{2}}{c(t)^{2}}
$$

where, $\mu_{0}$ denotes permeability of free space, $N$ is the number of turns of magnet coil, and $A$ denotes the area of 
the magnetic pole. For analytic purposes, a linearized model around the equilibrium point $\left(i_{0}, c_{0}\right)$ can be used.

$$
\begin{aligned}
& \Delta F(\Delta i(t), \Delta c(t))=-k_{i} \Delta i(t)+k_{c} \Delta c(t) \\
& \Delta \dot{i}(t)=\frac{k_{c}}{k_{i}} \Delta \dot{c}(t)-\frac{R}{L_{0}} \Delta i(t)+\frac{1}{L_{0}} \Delta v(t)
\end{aligned}
$$

where,

$$
L_{0}=\frac{\mu_{0} N^{2} A}{2 c_{0}}, k_{i}=\frac{\mu_{0} N^{2} A i_{0}}{2 c_{0}^{2}}, k_{c}=\frac{\mu_{0} N^{2} A i_{0}^{2}}{2 c_{0}^{3}}
$$

where, $v$ is voltage, and $R$ is resistance of coil.

\section{Yaw Motion Control for High-Speed Maglev Vehicle}

The purpose of the guidance control is to maintain the air gap and acceleration within an allowable range. Maintaining a constant air gap is critical to preventing a collision between the guideway and electromagnet. Reducing acceleration variation is crucial for passenger ride quality. The candidate variables in the control indicate absolute / relative positions and their derivatives. In this study, the following state feedback control law is employed.

$$
\Delta v(t)=k_{z a} \Delta \ddot{z}(t)+k_{z v} \Delta \dot{z}(t)+k_{z p} \Delta z(t)+k_{c v} \Delta \dot{c}(t)+k_{c p} \Delta c(t)
$$

The control law uses absolute position, absolute velocity, absolute acceleration, relative position (i.e., air gap), and relative velocity with appropriate gains.

\subsection{Five-state linear observer}

For practical purposes, all states in the feedback controller are not available. Hence, a linear state observer is used to estimate five states using acceleration and air gap measurements. Moreover, the observer can eliminate a DC bias and unwanted noises in its estimates. The control law with an observer is modified as follows [6].

$$
\Delta v(t)=k_{z a} \Delta \hat{\ddot{z}}(t)+k_{z v} \Delta \hat{\dot{z}}(t)+k_{z p} \hat{\Delta} z(t)+k_{c v} \Delta \hat{\dot{c}}(t)+k_{c p} \Delta \hat{c}(t)
$$

Estimates of the five states are utilized in the controller, and the linear observer is represented by the following.

$$
\begin{aligned}
& \dot{x}_{o}=A_{o} x_{o}+B_{o} u_{o} \\
& \dot{y}_{o}=C_{o} x_{o}+D_{o} u_{o} \\
& u_{o}=\left[\begin{array}{ll}
\Delta \ddot{z}(t) & \Delta c(t)
\end{array}\right]^{T}=\left[\begin{array}{ll}
\text { acc } & \text { gap }
\end{array}\right]^{T} \\
& y_{o}=\left[\begin{array}{lllll}
\Delta \hat{\ddot{z}}(t) & \Delta \hat{\dot{z}}(t) & \hat{\Delta} z(t) & \Delta \hat{\dot{c}}(t) & \Delta \hat{c}(t)
\end{array}\right]^{T}
\end{aligned}
$$

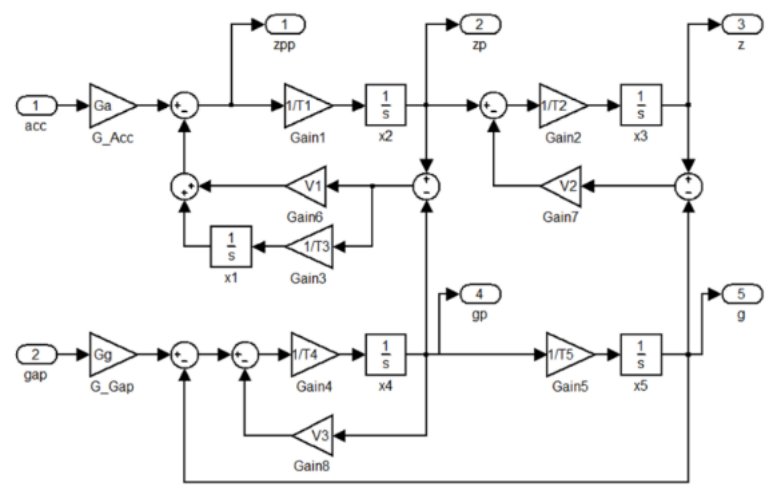

Fig. 5. Block diagram of the linear state observer

$$
\begin{aligned}
A_{o} & =\left[\begin{array}{ccccc}
0 & \frac{1}{T_{3}} & 0 & -\frac{1}{T_{3}} & 0 \\
-\frac{1}{T_{1}} & -\frac{V_{1}}{T_{1}} & 0 & \frac{V_{1}}{T_{1}} & 0 \\
0 & \frac{1}{T_{2}} & -\frac{V_{2}}{T_{2}} & 0 & \frac{V_{2}}{T_{2}} \\
0 & 0 & 0 & -\frac{V_{3}}{T_{4}} & -\frac{1}{T_{4}} \\
0 & 0 & 0 & \frac{1}{T_{5}} & 0
\end{array}\right], B_{o}=\left[\begin{array}{cc}
0 & 0 \\
\frac{1}{T_{1}} & 0 \\
0 & 0 \\
0 & \frac{1}{T_{4}} \\
0 & 0
\end{array}\right] \\
C_{o} & =\left[\begin{array}{ccccc}
-1 & -V_{1} & 0 & V_{1} & 0 \\
0 & 1 & 0 & 0 & 0 \\
0 & 0 & 1 & 0 & 0 \\
0 & 0 & 0 & 1 & 0 \\
0 & 0 & 0 & 0 & 1
\end{array}\right], D_{o}=\left[\begin{array}{cc}
1 & 0 \\
0 & 0 \\
0 & 0 \\
0 & 0 \\
0 & 0
\end{array}\right]
\end{aligned}
$$

where the constants are designed properly so that the observer possesses desired pass and stop bands. Implementation of the linear state observer is shown in Fig. 5.

In general, the observer can be designed separately from the controller. The control bandwidth of a stable suspension system is in the range $3-15 \mathrm{~Hz}$. The observer should then designed to have a faster response than the controller. Table 3 shows the parameters of the designed observer.

\subsection{Yaw motion control}

The applied voltage to the guidance magnet is determined according to the following:

$$
V_{\text {total }}=V_{\text {guide }}+V_{\text {yaw }}
$$

The term $V_{\text {guide }}$ is an individual guidance controller. The role of this controller is not only to guarantee the stability of the feedback system but also to maintain the air gap variation within an allowable deviation. Various control laws can be used to determine $V_{\text {guide }}$. The control law described in the previous section is employed as follows: 


$$
V_{\text {guide }}=k_{z a} \hat{\ddot{z}}+k_{z v} \hat{\dot{z}}+k_{z p}(\hat{z}-r)+k_{c v} \hat{\dot{c}}+k_{c p}(\hat{c}-r)
$$

where $r$ is a reference input that is set to a nominal air gap of $10 \mathrm{~mm}$.

The term $V_{\text {yaw }}$ is a yaw controller. The role of this controller is to minimize relative movement, especially with respect to bogie yaw motion. Various control laws can be used to determine $V_{\text {yaw }}$. The five state feedback control law is employed according to the following:

$$
V_{y a w}=k_{y z a} \hat{\ddot{z}}_{y}+k_{y z v} \hat{\dot{z}}_{y}+k_{y z p} \hat{z}_{y}+k_{y c v} \hat{\dot{c}}_{y}+k_{y c p} \hat{c}_{y}
$$

where $z_{y}$ and $c_{y}$ denote the differences between front and rear states.

Fig. 6 shows the block diagram of the guidance and yaw motion controls. For one bogie, guidance magnets are grouped into four sections corresponding to four corners. Two guidance magnets at each corner are controlled by the same command. Because guidance magnets are attached to a rigid body, left and right sides show the same motion.

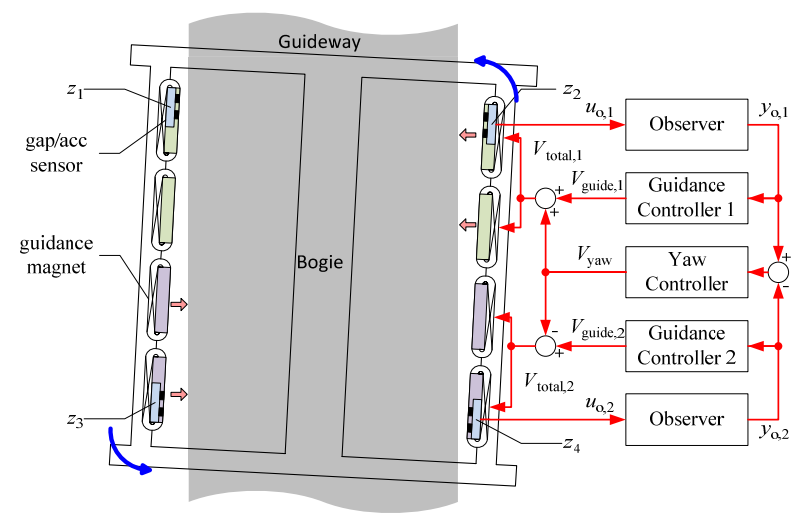

Fig. 6. Block diagram of guidance and yaw control

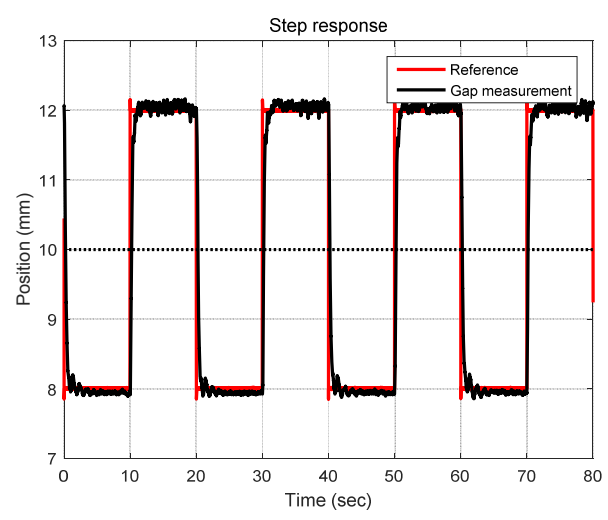

Fig. 7. Step response of guidance control

Table 3. Observer parameters

\begin{tabular}{c|c||c|c}
\hline Parameters & Values & Parameters & Values \\
\hline \hline$T_{1}$ & 0.1 & $T_{5}$ & 0.01 \\
\hline$T_{2}$ & 0.01 & $V_{1}$ & 2 \\
\hline$T_{3}$ & 0.1126 & $V_{2}$ & 0.09425 \\
\hline$T_{4}$ & 0.0001126 & $V_{3}$ & 0.1061 \\
\hline
\end{tabular}

Table 4. Controller gains

\begin{tabular}{c|c||c|c}
\hline \multicolumn{2}{c||}{ Guidance control } & \multicolumn{2}{c}{ Yaw control } \\
\hline Parameters & Values & Parameters & Values \\
\hline \hline$k_{z a}$ & 100 & $k_{y z a}$ & 40 \\
\hline$k_{z v}$ & 2000 & $k_{y z v}$ & 400 \\
\hline$k_{z p}$ & 0 & $k_{y z p}$ & 0 \\
\hline$k_{c v}$ & 2000 & $k_{y c v}$ & 400 \\
\hline$k_{c p}$ & 300 & $k_{y c p}$ & 12 \\
\hline
\end{tabular}

Hence, the coordinates of both sides are closely related as follows:

$$
\begin{aligned}
& z_{\text {front }}=z_{1}=z_{2}, c_{1}+c_{2}=\text { const } . \\
& z_{\text {rear }}=z_{3}=z_{4}, c_{3}+c_{4}=\text { const } .
\end{aligned}
$$

The sensor signals can be shared by left and right sides because of the aforementioned symmetry. Guidance magnets utilize attractive forces. Therefore, a pair of magnets with a wider air gap is active.

\section{Experimental Results}

To verify the proposed control algorithm, experiments were conducted using the full-scale maglev test vehicle on a test track, as shown in Fig. 1. Table 4 shows the gains of the designed controller. The control parameters were tuned in order to maximize the control bandwidth. In experiments, the control gains $\left(k_{z p}, k_{y z p}\right)$ associated with absolute positions were not used because the guideway deviation may result in the steady state error in the air gap response. Velocity (or derivative) gains were set to be the same to achieve similar damping effects.

Before verifying the effect of yaw motion control, in order to see the transient response of the proposed individual guidance controller, we measured the step response and plotted it in Fig. 7. As shown, the proposed individual guidance control provides rapid response characteristics and a sufficient damping ratio. The rise time of the response is $429 \mathrm{~ms}$ and maximum overshoot is $2 \%$. The expected control bandwidth is about $2.1 \mathrm{~Hz}$.

To verify the effect of the proposed yaw motion controller, control performance was examined with and without the yaw controller. After levitation, a reference signal employing a $3 \mathrm{~mm}$ amplitude and a $1 \mathrm{~Hz}$ frequency was intentionally excited to the front guidance magnet. This reference signal resulted in intentional bogie yaw motion. Fig. 8 shows the results of the experiments with and without the yaw controller. As shown, because of the yaw motion control, the relative movement of the guidance magnets was significantly reduced by $67 \%$.

\section{Conclusion}

Additional guidance electromagnets are necessary to 

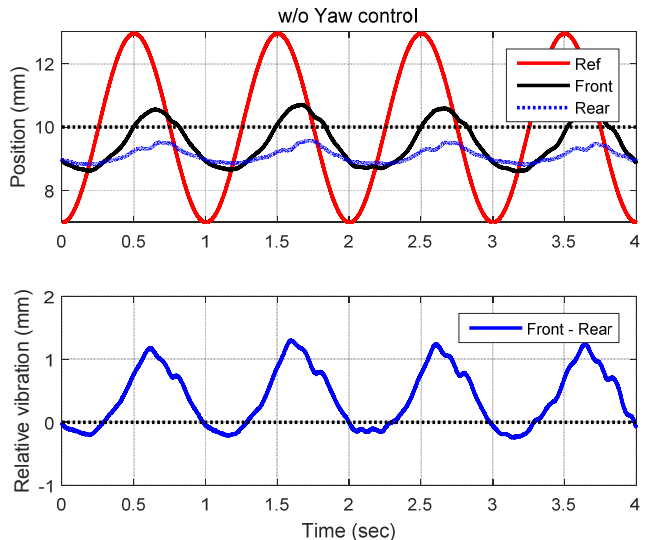

(a)
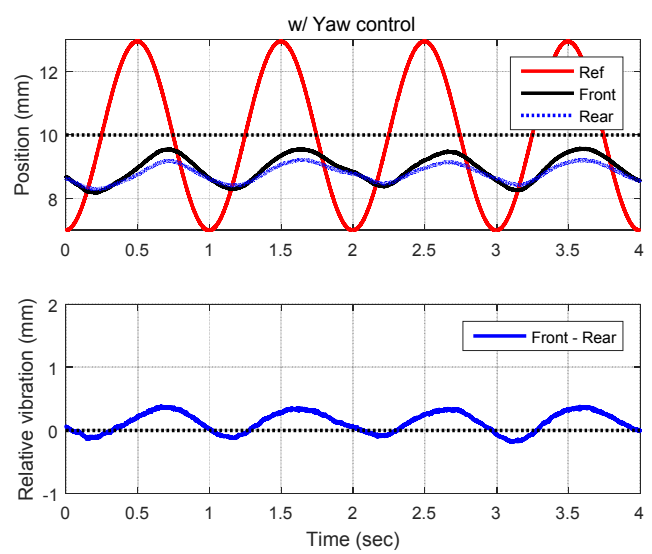

(b)

Fig. 8. Effect of the yaw motion control (a) without yaw motion control and (b) with yaw motion control

maintain the lateral air gap within an allowable range. First, we designed a U-shaped electromagnet using transverse flux. Many aspects were considered in the design process and characteristics of the final design were verified with finite element analysis. Second, bogie yaw motion was incorporated into the guidance control law to reduce variations in both the airgap and bogie yaw angle. In an externally excited bogie yaw motion, the effect of the proposed yaw control was verified by experiments using a full-scale maglev vehicle. From the results, we verified that because of the proposed yaw control, the relative movement of the guidance magnets, especially in bogie yaw motion, can be reduced by $67 \%$.

\section{References}

[1] H.W. Lee, K.C. Kim, and J. Lee, "Review of maglev train technologies," IEEE Trans. Magn., vol. 42, no. 7, pp. 1917-1925, 2006.

[2] J. Lim, C.H. Kim, J.B. Han, and H.S. Han, "Design of an electromagnet with low detent force and its control for a maglev super-speed vehicle," J. Elect.
Eng. Technol., vol. 10, no. 4, pp. 1668-1674, 2015.

[3] K. Heinrich and R. Kretzschmar, Transrapid MagLev System, Hestra-Verlag Darmstadt, Germany, pp. 7677, 1989.

[4] A. Hao, Z. Long, and W. Chang, "Design of the robust controller of the guidance system in highspeed maglev Train," Journal of the China Railway Society, vol. 30, no. 6, pp. 40-45, 2008.

[5] C.H. Kim, K.J. Kim, J.S. Yu, H.W. Cho, "Dynamic performance evaluation of 5-DOF magnetic levitation and guidance device by using equivalent magnetic circuit model," IEEE Trans. Magn., vol. 49, no. 7, pp. 4156-4159, 2013.

[6] C.H. Kim, J. Lim, J.M. Lee, H.S. Han, D.Y. Park, "Levitation control design of super-speed maglev trains," World Automation Congress 2014, Kona, HI, USA, 2014.

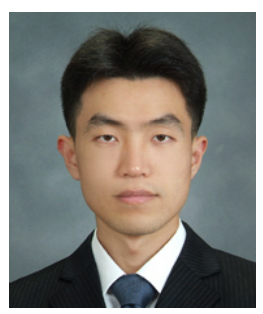

Chang-Hyun Kim He received the B.S., M.S., and Ph.D. degrees in electrical and electronic engineering from Korea Advanced Institute of Science and Technology in 2000, 2002 and 2009, respectively. Since 2009, he has been a senior researcher in KIMM (Korea Institute of Machinery and Materials). His research interest is the control of magnetic levitation and linear propulsion systems.

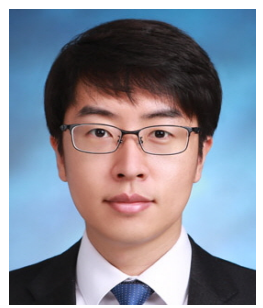

Chang-Wan Ha He received the B.S. degree in mechanical engineering from Handong Global University, Pohang, Korea, in 2008 and the M.S. and Ph.D. degrees in mechanical engineering from KAIST (Korea Advanced Institute of Science and Technology), Daejeon, Korea, in 2010 and 2014, respectively. Since 2014, he has been a senior researcher in KIMM (Korea Institute of Machinery and Materials). His research interest is the control of magnetic levitation systems and motion control including input shaping technique.

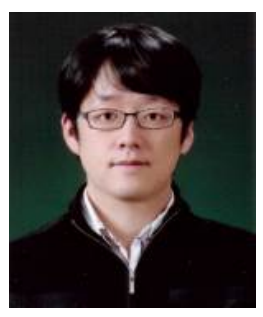

Jaewon Lim He received the B.S., M.S., and Ph.D. degrees in electrical and computer engineering from Seoul National University in 2004, 2006 and 2011, respectively. Since 2011, he has been a senior researcher in KIMM (Korea Institute of Machinery and Materials). His research interest is the design of magnetic actuator and linear propulsion systems. 


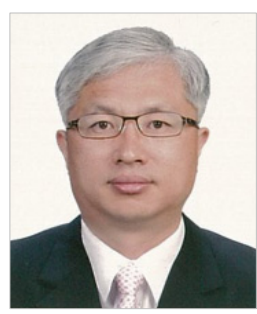

Hyung-Suk Han He received the Ph.D. degree in mechanical engineering from Ajou University, Suwon, Korea, in 1997. Since 1997, he has been a principal researcher in KIMM (Korea Institute of Machinery and Materials). His research interest is the dynamic simulation of maglev vehicles. He developed the ECOBEE, urban maglev vehicle, and SUMA550, highspeed maglev test vehicle. Based on the 20-year experience in maglev vehicle technology, he published an academic book titled on 'Magnetic Levitation' in 2016.

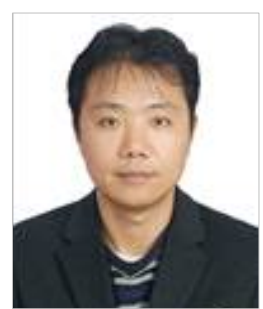

Ki-Jung Kim He received his B.S., M.S., and Ph.D. degrees in Mechatronics Engineering from Chungnam National University, Daejeon, Korea, in 2009, 2011 and 2014, respectively. $\mathrm{He}$ is currently a research fellow in Research Institute for High-Technology Transportation at Chungnam National University, Daejeon, Korea His research interests are the computational mechanics in the human body and vehicles. 